\title{
A new p.(Ile66Serfs*93) IGF2 variant is associated with pre- and postnatal growth retardation
}

\author{
Denise Rockstroh ${ }^{1,2}$, Heike Pfäffle ${ }^{1,2}$, Diana Le Duc ${ }^{3}$, Franziska Rößler ${ }^{3}$, Franziska Schlensog-Schuster ${ }^{1}$, \\ John T Heiker ${ }^{4}$, Jürgen Kratzsch ${ }^{5}$, Wieland Kiess ${ }^{1,2}$, Johannes R Lemke ${ }^{3}$, \\ Rami Abou Jamra ${ }^{3}$ and Roland Pfäffle ${ }^{1,2}$
}

${ }^{1}$ Department of Women and Child Health, University of Leipzig Hospitals and Clinics, Leipzig, Germany, ${ }^{2}$ Center for Pediatric Research Leipzig, Department of Women's and Child Health, University Hospital for Children \& Adolescents, University of Leipzig, Leipzig, Germany, ${ }^{3}$ Institute of Human Genetics, University of Leipzig Hospitals and Clinics, Leipzig, Germany, ${ }^{4}$ Institute of Biochemistry, Faculty of Life Sciences, Pharmacy and Psychology, University of Leipzig, Leipzig, Germany, and ${ }^{5}$ Institute of Laboratory Medicine, Clinical Chemistry and Molecular Diagnostics, University Hospital Leipzig, Leipzig, Germany

Correspondence should be addressed to D Rockstroh Email Denise.Rockstroh@medizin. uni-leipzig.de

\begin{abstract}
Objective: The IGF/IGF1R axis is involved in the regulation of human growth. Both IGF1 and IGF2 can bind to the IGF1R in order to promote growth via the downstream PI3K/AKT pathway. Pathogenic mutations in IGF1 and IGF1R determine intrauterine growth restriction and affect postnatal body growth. However, to date, there are only few reports of pathogenic IGF2 mutations causing severe prenatal, as well as postnatal growth retardation.

Results: Here we describe a de novo c.195delC IGF2 variant (NM_000612, p.(Ile66Serfs*93)) in a 4-year-old patient with severe pre- and postnatal growth retardation in combination with dystrophy, facial dimorphism, finger deformities, as well as a patent ductus. Cloning and sequencing of a long-range PCR product harboring the deletion and a SNP informative site chr11:2153634 (rs680, NC_000011.9:g.2153634T>C) demonstrated that the variant resided on the paternal allele. This finding is consistent with the known maternal imprinting of IGF2. 3D protein structure prediction and overexpression studies demonstrated that the p.(Ile66Serfs*93) IGF2 gene variation resulted in an altered protein structure that impaired ligand/receptor binding and thus prevents IGF1R activation.

Conclusion: The severity of the phenotype in combination with the dominant mode of transmission provides further evidence for the involvement of IGF2 in growth disorders.
\end{abstract}

\section{Introduction}

Insulin-like growth factors (IGFs) belong to the GH/IGF axis, a highly evolutionarily conserved network that regulates human growth (1). The system is composed of two related growth factors (IGF1 and IGF2), their cell-surface receptors (IGF1R and IGF2R), and IGF-binding proteins (IGFBPs), which increase functional stability of IGFs (2). In contrast to IGF1, which is produced in response to growth hormone $(\mathrm{GH})$ stimulation in hepatic and non-hepatic tissues thereby regulating postnatal growth $(3,4)$; IGF2 is expressed during embryonic and fetal development in a wide variety of somatic tissues with highest expression in the fetal liver and placenta (3). IGF2 was one of the first genes shown to be imprinted resulting in a transcription mainly from the paternal allele, whereas the maternal allele is silenced (5). Many of the effects that IGF2 has on growth have been deduced from the clinical phenotypes of the Beckwith-Wiedemann syndrome (BWS) and Silver-Russell syndrome (SRS), where IGF2 gene imprinting is disrupted.

Published by Bioscientifica Ltd. 
The BWS is caused by a biallelic IGF2 expression, leading to fetal overgrowth and an increased risk for childhood cancer (6). The SRS phenotype is characterized by intrauterine growth retardation, short stature, skeletal asymmetry and clinodactyly (7). In app. 10\% of the cases, SRS is associated with a maternal uniparental disomy on chromosome 7 (UPD7), while 30-50\% of the cases are associated with hypomethylation of the parental imprinting center region 1 (ICR1) on chromosome 11p15.5 (8). So far only few heterozygous, paternally inherited IGF2 mutations have been identified in humans with evidence of pathogenicity. Begemann et al. reported the first heterozygous IGF2 variation (NM_001127598: c.191C $\rightarrow$ A, p.Ser64Ter) in a multigenerational family with a severe growth retardation (9). Habib et al. identified a heterozygous missense variation (NM_000612: c.78C >G, p.Tyr26Ter) (10), while Yamoto et al. identified an indel variation (NM_000612: c.110_117delinsAGGTAA， p.(Leu37Gln $\left.f s^{*} 31\right)$ ) (11) in patients with a SRS-like phenotype. These three mutations satisfied the condition for the occurrence of nonsensemediated mRNA decay. In addition, Liu et al. and Habib et al. identified novel heterozygous missense variants (NM_000612, c.101G>A, p.Gly34Asp; c.158-159dup, p.(Arg54Alafs*7)), which might influence IGF2/IGF1R interaction $(10,12)$. All mutations are located on the paternal allele.

In this study, we present the case of a 4-year-old boy with a heterozygous de novo IGF2 frameshift variation (NM_000612: c.195delC, p.(Ile66Ser $\left.f s^{*} 93\right)$ ) on the paternal allele, which is associated with severe pre- and postnatal growth retardation. In contrast to previous frameshift mutations, the c.195delC variation leads to the expression of a mutant IGF2 protein.

\section{Subjects and methods}

\section{Patient description}

The index patient (patient III.2, Fig. 1A) is the first child of a non-consanguineous Caucasian couple. The parents were clinically healthy and of normal stature (paternal height: $180.0 \mathrm{~cm},-0.10$ SDS; maternal height: $170.0 \mathrm{~cm}, 0.29$ SDS). Poor fetal growth was first noted in the 28th week of gestation; whereby, amniocentesis revealed a normal 46, XY karyotype. The patient was delivered spontaneously at $39+1$ weeks of gestation with a birth weight of $1485.0 \mathrm{~g}$ ( $-5.00 \mathrm{SDS})$, a birth length of $39.0 \mathrm{~cm}(-5.22$ SDS) and a head circumference of $32.0 \mathrm{~cm}(-2.50$ SDS). Immediately after birth, the patient showed respiratory distress and was therefore admitted to the neonatal intensive care unit. He required oxygen supplementation and continuous positive airway pressure for 2 weeks. He also had a cleft-palate leading to feeding difficulties. At the age of 3.4 years, he was presented to our pediatric endocrine department with the clinical picture of severe growth retardation in combination with a pronounced underweight (height: $77.3 \mathrm{~cm}$ (height SDS: -5.41); weight: $7.3 \mathrm{~kg}$ (BMI: $12.2 \mathrm{~kg} / \mathrm{m}^{2}$; BMI SDS: -3.51)) (Fig. $1 \mathrm{~B}$ and C I-II). He showed a low height velocity of $5.1 \mathrm{~cm} /$ year (-2.98 SDS) and his bone age (Tanner-Whitehouse 3) was retarded by approximately 1.7 years (Fig. 1B). Although his head circumference was small (-2.36 SDS), he had frontal bossing and gave the impression of macrocephaly relative to his height ( $-5.40 \mathrm{SDS}$ ) (Fig. 1C III-V). Other syndromic features included a long philtrum, high forehead, retrognathia and a clinodactyly (Fig. 1C VIVIII). Additionally, a patent ductus arteriosus which closed spontaneously, and a penoscrotal transposition, which was surgically corrected together with the cleftpalate were observed. Although he learned to walk and speak relatively late (at about 24 months), his intelligence appears to be normal (measured with the K-ABC test (13)). At the age of 4.7 years, his height was $84.5 \mathrm{~cm}$ (height SDS: -5.36; Fig. 1B) and his weight $9.0 \mathrm{~kg}$ (BMI: $12.6 \mathrm{~kg} / \mathrm{m}^{2} ; \mathrm{BMI}$ SDS: -2.66). At this time, serum IGF1 levels $(110.9 \mu \mathrm{g} / \mathrm{L}$, $-0.23 S D S$ ) were normal, while IGF2 and IGFBP3 serum concentrations $(210 \mathrm{ng} / \mathrm{mL} ; 1.69 \mathrm{mg} / \mathrm{L},-2.23 \mathrm{SDS})$ were markedly decreased (Table 1). At the age of 5.24 years recombinant human GH therapy $(0.022 \mathrm{mg} / \mathrm{kg} / \mathrm{d})$ was initiated and on his latest examination (5.5 years) his height was $91.2 \mathrm{~cm}$ (height SDS: -4.83) and his weight 9.6 kg (BMI: $11.5 \mathrm{~kg} / \mathrm{m}^{2}$; BMI SDS: -4.02). There had been no similar cases within the family and the patient has one older half-brother who is reported to be healthy and of normal stature. This study was approved by the Ethics Committees of the University of Leipzig.

\section{Patient consent}

Informed consent has been obtained from the patient's guardian for publication of the case report, accompanying images and DNA analyses, which is in line with the Declaration of Helsinki.

\section{Identification and parental origin of the variant}

Next-generation sequencing (NGS) analyses were performed on genomic DNA extracted from whole blood of the affected patient and parents. Enrichment for 4813 genes (TruSight One, Illumina, San Diego, CA, USA) 
A

I

II

III

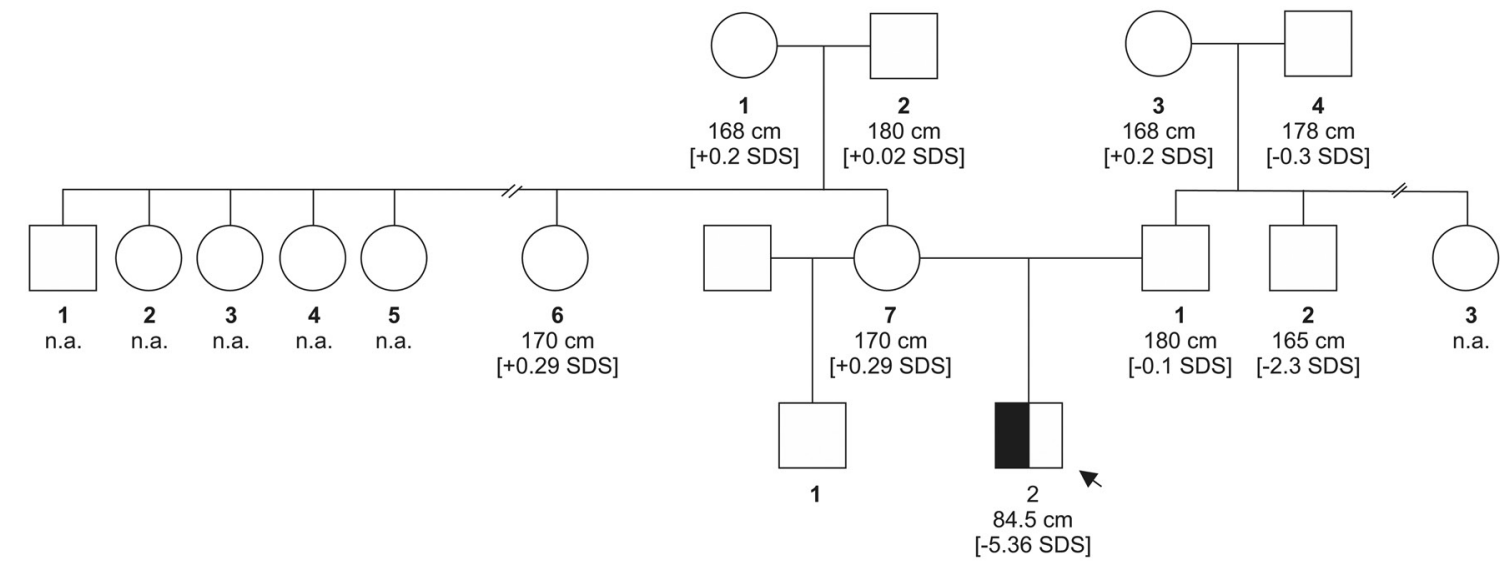

B

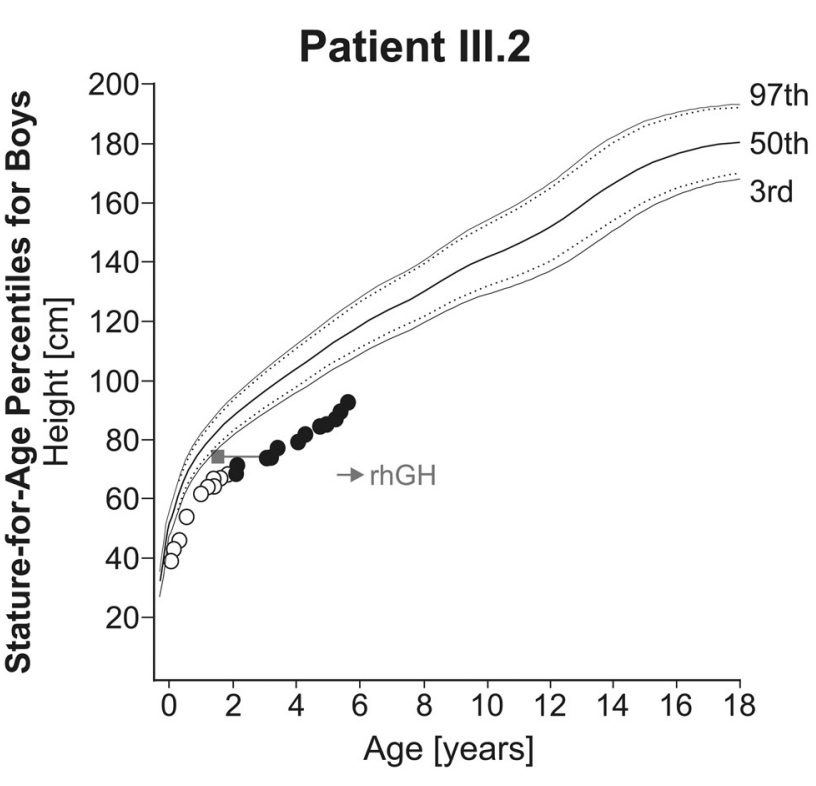

C

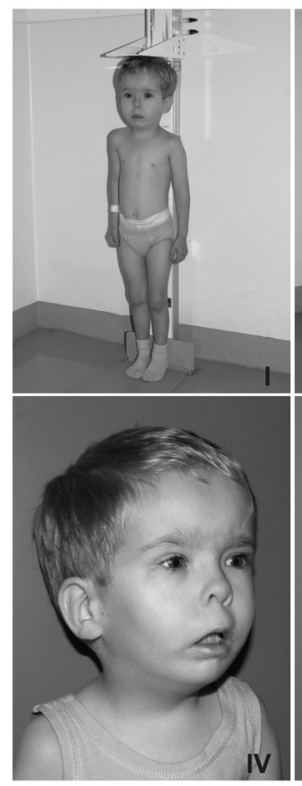

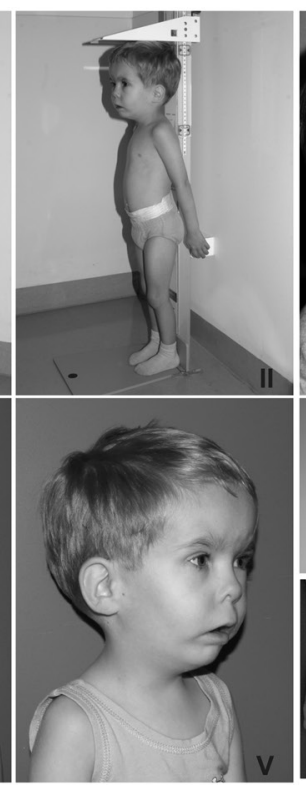

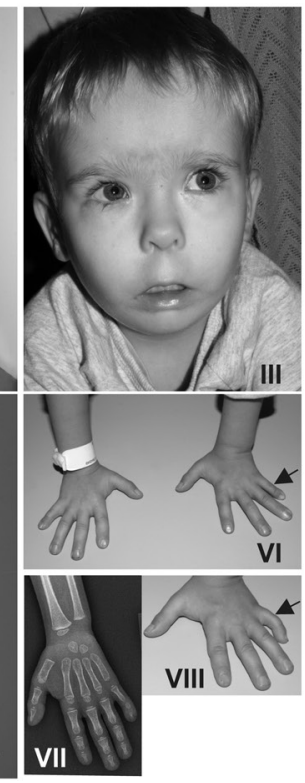

\section{Figure 1}

Pedigree, growth curve and clinical characteristics of the patient. (A) The index patient (III.2) bearing the heterozygous p.(Ile66Serfs*93) variation is indicated by a half-filled symbol and marked by an arrow. Height (cm) and standard deviation score (SDS) of the last reported visit is shown below the symbol. Crossed out lines indicate half siblings (n.a., no data available). Growth curve of the index patient $(B)$ indicated a severe growth failure. Bone age is shown by a gray square, while initiation of rhGH therapy is shown by a gray arrow. White circles indicated patients' height adjusted for week of pregnancy, while black circles indicate unadjusted values. (C) Clinical features of the index patient: short stature (I, II), triangular face (III), low-set ears (IV, V), frontal bossing (V) and a clinodactyly (VI-VIII marked by an arrow).

was performed following the manufacturer's protocol. Sequencing was performed on NextSeq 550 as given by the manufacturer at the Institute of Human Genetics, Leipzig, Germany. Mean depth of coverage for the analysis was $121 \times$ and quality threshold $(\geq 20 \times)$ was achieved for $95.5 \%$ of the target sequence. Identified variants were annotated using standard databases and were filtered based on the established criteria using the Varvis software (Limbus, Rostock, Germany). The identified IGF2 variant was confirmed by Sanger sequencing using the amplification oligos: F: 5'-CAGGGGTGGTTTGCTCC-3' and R: 5'-AAGTCAC GGGTCCTTGTCC-3'.

Allele specific amplification: Next, we examined whether the IGF2 variant occurred on the paternal 


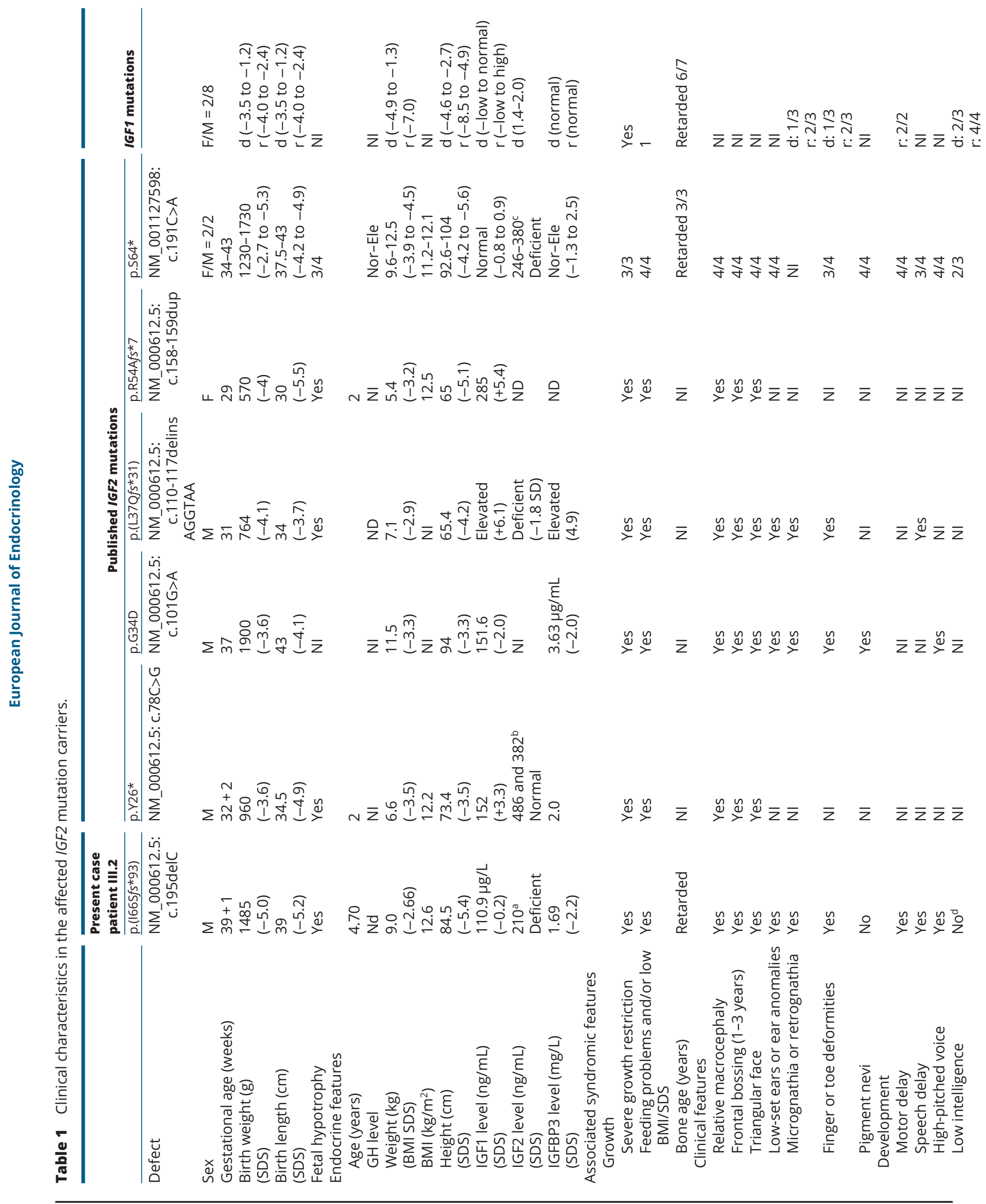




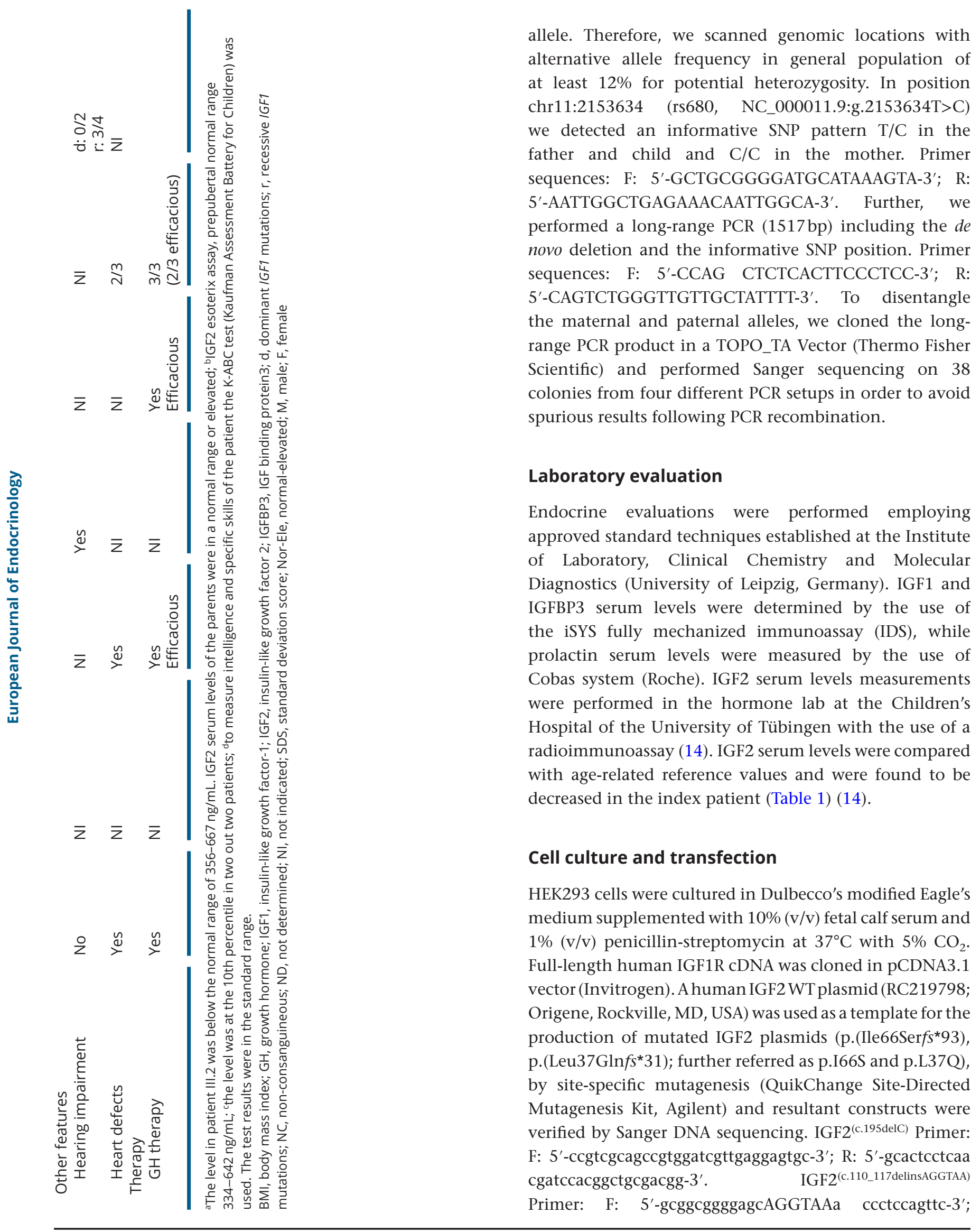


R: $\quad$ 5'-gaactggagggtTTACCTgctcccoccgc-3'. Transient transfections were performed in 6-well plates using TurboFect reagent (Thermo Scientific). Analysis of transient expression was performed $48 \mathrm{~h}$ after transfection.

\section{Western blot analysis}

For stimulation experiments, cells were washed and serum-starved for $2 \mathrm{~h}$ prior treatment with recombinant, human IGF1 (10 nM, MBL, Woburn, MA, USA). Total cell lysates were collected $30 \mathrm{~min}$ post treatment and wholecell lysates were separated on 10\% SDS-polyacrylamide gels (15\% for IGF2 proteins), transferred to nitrocellulose membranes and probed with anti-IGF2 (1:500; \#ABC504, Millipore), anti-pIGF1R (1:500, \# 3024S, Cell Signaling), anti-IGF1R (1:500, \# 3018S, Cell Signaling) or anti- $\beta$ actin antibodies (1:5000, sc-58673, Sigma) as indicated. Secondary HRP-conjugated anti-mouse IgG or antirabbit IgG antibodies, appropriate SuperSignal substrates (Thermo Fisher Scientific), and GE Healthcare's Hyperfilm ECL were used for chemiluminescence visualization.

\section{Structural model for the $\mathrm{p} .166 \mathrm{~S}$ variant}

The impact of the IGF2 variant on protein folding and structure was assessed by protein 3D structure prediction using Iterative Threading ASSEmbly Refinement (I-TASSER) (15) using the recently reported crystal structure of IGF2 (PDB id: 1IGL) as structural template (16). Figures of protein structures were prepared using PyMOL (www. pymol.org).

\section{Statistical analysis}

Data are presented as mean \pm s.E.M. of at least three independent experiments. Differences were analyzed by a one-way ANOVA with Turkey's test for multiple comparisons. Statistical analysis of the data was performed using GraphPad Prism 5 software.

\section{Results}

\section{Identification of a new p.I66S IGF2 mutation}

The index patient was likely to have a diagnosis of SRS based on a clinical scoring system (17). Given that SRS can be caused by maternal UPD7 or hypomethylation of the parental ICR1/ICR2 region (8), we performed single-nucleotide primer extension and multiplex ligation-dependent probe amplification analyses. We found no evidence of a UPD7 or epimutations in the area of ICR1/ICR2. However, NGS sequencing revealed a heterozygous c.195delC deletion in IGF2 (NM_000612: c.195delC, p.(Ile66Ser $f s^{\star 93}$ ); further referred as p.I66S), which was confirmed by Sanger-based sequencing analysis (Fig. 2A). Both parents do not carry the deletion (paternity was verified). The mutation is not listed in any of the large-scale variant databases and is predicted to be pathogenic or likely pathogenic (Table 2). No other rare variant was identified in genes known to be involved in growth failure, including IGF1, IGF1R, IGFALS, IGFBP3, PLAG1 or HMGA2.

The c.195delC deletion occurred in exon 3 of the coding sequence and induces a frameshift leading to an early stop codon at position 93 (Fig. 2B). So far, only five further human IGF2 mutations have been identified with evidence of pathogenicity. Three heterozygous nonsense substitutions in exon 8 (p.Y26*, p.G34D, p.(L37Q $\left.f s^{*} 31\right)$ ) and two substitutions in exon 9 (p.(R54A $\left.f s^{\star} 7\right)$, p.S64*) were identified in patients with SRS-like phenotype (Fig. 2C). All mutations resided on the paternal allele. To show that the p.I66S IGF2 variant is located on the paternal allele we first identified the rs680 (NC_000011.9:g.2153634T>C) informative SNP pattern: father heterozygous $\mathrm{T} / \mathrm{C}$, mother homozygous $\mathrm{C} / \mathrm{C}$ and index heterozygous $\mathrm{T} / \mathrm{C}$. We then performed long PCR amplification in the index for a 1504-bp region spanning the pathogenic variant and rs680 (NC_000011.9:g.2153634T>C). Finally, we cloned the PCR product and carried out Sanger sequencing of the clones. This revealed a segregation of the pathogenic variant (one base pair) deletion with the $\mathrm{T}$ allele, showing that the de novo variant occurred on the paternal allele.

\section{The p.I66S variant influences IGF2 protein structure}

In silico surveys revealed the novelty and putative damaging impact of the p.I66S IGF2 variation, which prompted us to assess the protein structure. IGF2 is synthesized as an inactive 180-aa precursor protein, which after posttranslational cleavage constitutes the bioactive core IGF2 protein (67-aa) (18) (Fig. 3A). There are two crucial aa-cleavage sites: TQRLRR ${ }^{104}$ and PAKSER ${ }^{68}$ (19). The p.I66S variation induces a frameshift which alters both cleavage sites (Fig. 3B). 3D protein structure determination illustrates the putative altered p.I66S IGF2 protein, which displays the extended and likely unstructured nonsense C-terminal residues of the mutant protein compared to the WT (Fig. 3C). 
A

\section{Wild-type}

DNA-sequence

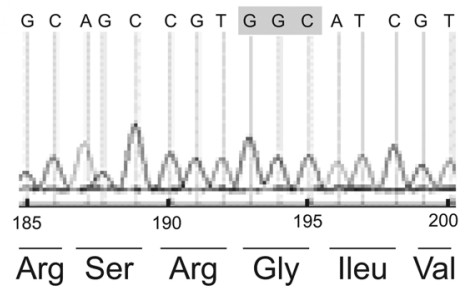

Index Pateint

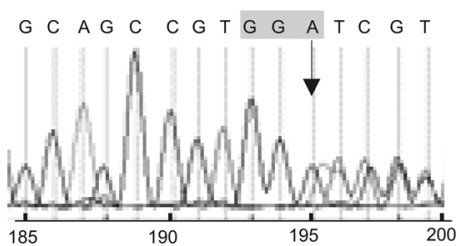

aa-sequence

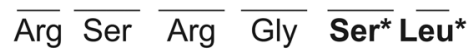

C

IGF2

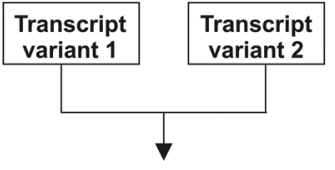

92

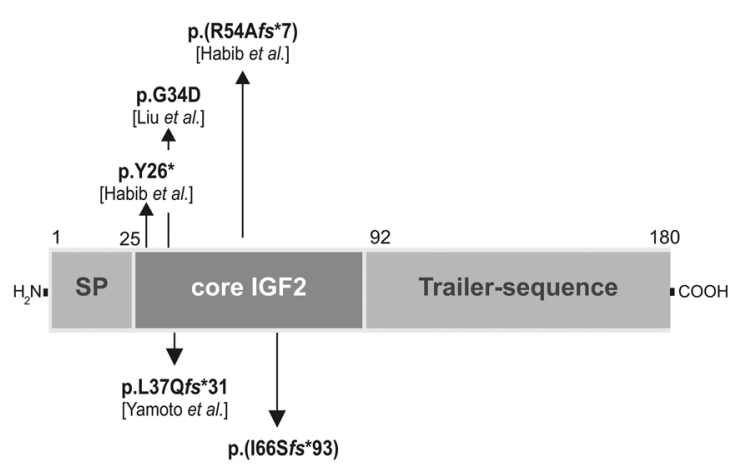

B

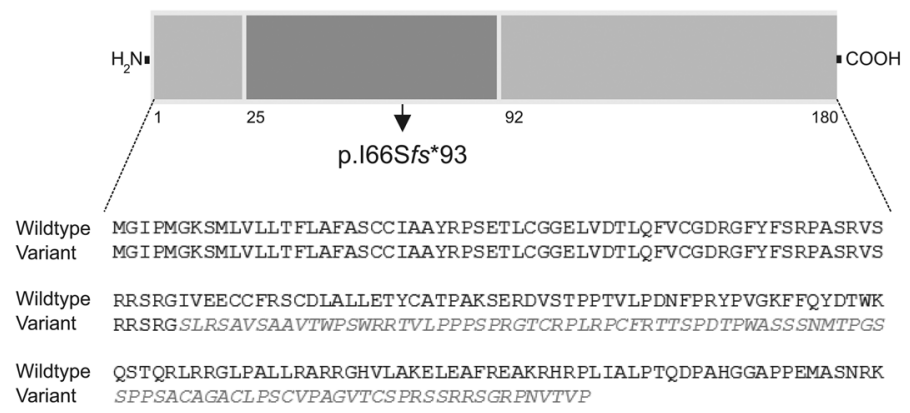

IGF2

Isoform 2 (prepro)

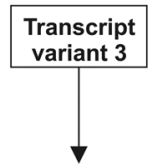

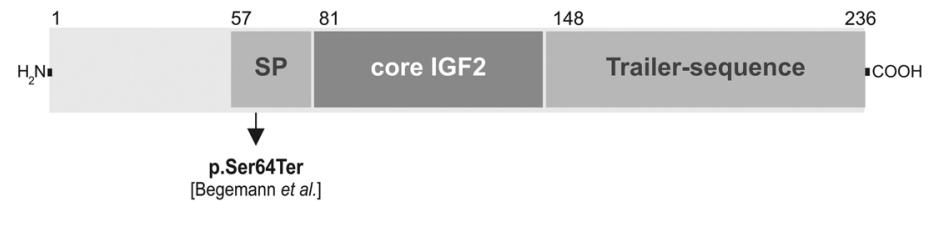

\section{Figure 2}

Identification of a novel p.(Ile66Serfs*93) IGF2 variation. (A) Electropherograms of Sanger sequencing analyses. Arrow, position of the c.195delC deletion; *frameshift of the aa sequence. (B) Structure of the IGF2 protein. Normal protein translation generates a 180-aa IGF2 precursor protein. The I66S variation is predicted to results in a frameshift (position 66) in combination with a premature stop codon at position 93. The changed aa sequence in the mutant beginning from position 66 is shown in italics. (C) Schematic illustration of so far identified IGF2 mutations $(9,11,12)$. IGF2 encode an inactive 180-aa (Isoform 1, NP_000603) or 236-aa (Isoform 2, NP_001121070) precursor protein that includes a 24-aa signal peptide (SP), a 67-aa core IGF2 and a 89-aa trailer sequence (18). The p.I66S variation is localized in the core-IGF2 sequence of isoform 1 (NM_000612).

\section{The IGF2 mutant prevents IGF1R activation probably through an impaired ligand/receptor interaction}

We next addressed whether the p.I66S variant is secreted by cells or bound to intracellular degradation. Therefore, p.I66S and WT plasmids were generated (Fig. 3D). The results show that WT and mutant IGF2 plasmids were expressed and secreted by HEK293 cells, and protein levels in supernatants were comparable to the WT (Fig. 3D). Both IGF1 and IGF2 exert their growth promoting effect through the interaction with IGF1 receptor (IGF1R). Ligand/receptor complexing leads to IGF1R phosphorylation (pIGF1R), which activates the PI3K/ AKT pathway regulating cell proliferation, differentiation and survival (4). In HEK293 cells, IGF1 induces pIGF1R in a dose-dependent manner with highest activation at a concentration of $100 \mathrm{nM}$ (Fig. 3E), which was also seen after stimulation with IGF2-WT supernatant (Fig. 3F). Next, we investigated the capacity of the p.I66S variant to activate IGF1R, whereby an empty vector and the 
Table 2 In silico analyses of IGF2 variants.

\begin{tabular}{|c|c|c|c|c|}
\hline \multirow[b]{2}{*}{ Variant } & \multicolumn{3}{|c|}{ Position } & \multirow[b]{2}{*}{ Prediction $^{d}$} \\
\hline & Genomic & Coding (exon) & $\underline{\text { Protein }^{c}}$ & \\
\hline $1^{\mathrm{a}}$ & g.25764 & c. $78 \mathrm{C}>\mathrm{G}(8)$ & p.Tyr26* & $P$ \\
\hline $2^{a}$ & g.25787 & c.101G >A (8) & p.Gly34Asp & LP \\
\hline $3^{a}$ & g.25796_25803 & c.110_117delinsAGGTAA (8) & p.(Leu37GInfs*31) & $P$ \\
\hline $4^{a}$ & g.27545_27546 & c.158-159dup (9) & p.(Arg54Alafs*7) & $\mathrm{P}$ \\
\hline $5^{a}$ & g.27582_27582 & c.195delC (9) & p.(Ile66Serfs*93) & $\mathrm{P}$ \\
\hline $6^{b}$ & g.25709 & c.191C>A (9) & p.Ser64Ter & LP \\
\hline
\end{tabular}

aNM_000612; bNM_001127598; ' CUniProtKB P01344; dIGF2 variations are predicted to be pathogenic (P) or likely pathogenic (LP) according to the standards and guidelines of the American College of Medical Genetics and Genomics and the Association for Molecular Pathology (ACMG-AMP); all in silico analysis performed in August 2018.

previously identified p.(Leu37Gln $\left.f s^{*} 31\right)$ frameshift mutation (further referred as p.L37Q) (11) were used as controls. The IGF2-WT robustly activates IGF1R after IGF1R/IGF2 co-transfection, while the p.I66S mutant showed an impaired activation capacity (Fig. 4A and B). In accordance with the predicted nonsense-mediated mRNA decay for the p.L37Q mutant, no mutant IGF2 was detected (Fig. 4A and B), which consequently diminished IGF1R activation (Fig. 4A and B). Similar results were obtained when IGF1R transfected cells were treated with the IGF2-WT or IGF2-MUT supernatants (Fig. 4C and D). These findings indicate that the p.I66S IGF2 variant is nonfunctional. At this point the question arises to what extent the mutant is able to interact with the IGF1 receptor. Currently, the understanding of the IGF2/IGF1R interaction is lacking chiefly due to unavailability of any structural data. However, sequence and structural similarities to the insulin/insulin receptor complex suggest that IGF2 may have similar binding surfaces. The previously known binding epitopes of the IGF1R domains L1, CR, L2, (F1-F2)' are shown in Fig. 4E according to Vashisth et al. (20). Although most binding residues remain intact in the mutant, the V43, D52, L53, E57, T58, T62, P63 and A64 residues are missing, which might affect ligand/receptor interaction. To test this, we co-transfected IGF1R and IGF2 constructs and measured IGF1R activation after IGF1 stimulation (Fig. 4F). Both IGF1R/IGF2-WT and IGF1R/IGF2-p.I66S co-transfected cells showed an increased activation after stimulation, indicating that we can rescue IGF1R activation in IGF1R/ IGF2-p.I66S co-transfected cells.

\section{Discussion}

Here, we present the case of a de novo paternal p.(Ile66Ser $\left.f s^{*} 93\right)$ frameshift mutation in the IGF2 gene, which is associated with a SRS-like phenotype. Functional analyses indicated that the IGF2 variant lost its ability to interact with the IGF1R due to changes in protein structure, which leads to only marginal activation of IGF1R. The results of this study in combination with previous reports indicate that the IGF2 variation might be responsible for the pathogenic phenotype of the patient.

IGFs are important for adequate growth and disruption of $I g f 1$ or $I g f 2$ in mice leads to a reduction in birth weight and size. Heterozygous $I g f 1^{(+-)}$mutants had a birth weight 10-20\% less than WTs, while homozygous Ig $f 1^{(-/-)}$mutants possess a birth weight $40 \%$ less than WTs, along with underdeveloped muscles and lungs, and high perinatal mortality $(21,22)$. In humans, homozygous IGF1 mutations are associated with severe intrauterine growth restriction (IUGR), postnatal growth failure and developmental delay (23). In contrast, homozygous Igf $2^{(-/-)}$ and heterozygous mutant mice carrying a paternally derived mutated $I g f 2$ gene $\left(I g f 2^{(\mathrm{p}-)}\right)$ are phenotypically indistinguishable. They exhibited a $60 \%$ reduction in birth weight compared to WT littermates, but were otherwise viable and fertile (24). These results indicate that IGF2 is a major contributor to intrauterine growth in mice, which is supported by the finding that IGF2 concentrations are more than six time higher than IGF1 concentration in human cord blood (25). For long, no human IGF2 mutation was reported, leading to the assumption that IGF2 is essential for human intrauterine survival. Recently five paternally derived IGF2 mutations (p.Tyr26Ter (10), p.Gly34Asp (12), p.(Leu37Glnf $\left.s^{*} 31\right)(11)$, p. $\left(\operatorname{Arg} 54 A l a f s^{*} 7\right)$ (10) and p.Ser64Ter (9)) have been described with evidence of pathogenicity in patients with an SRS-like phenotype. The clinical characteristics of all patients are very similar. A comparison reveals that all patients exhibited an IUGR, which is in line with murine studies (24). At this point it is unclear whether this is a direct or indirect effect, given that IGF2 contributes to placental size, morphology and nutrient delivery affecting the efficiency with which the placenta supports fetal growth (26). Furthermore, 
A

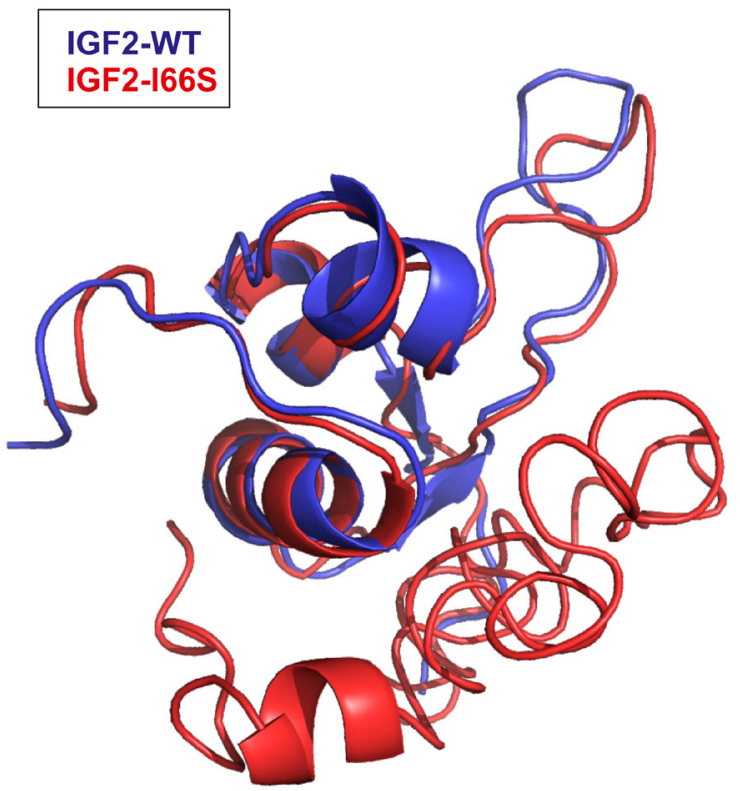

D
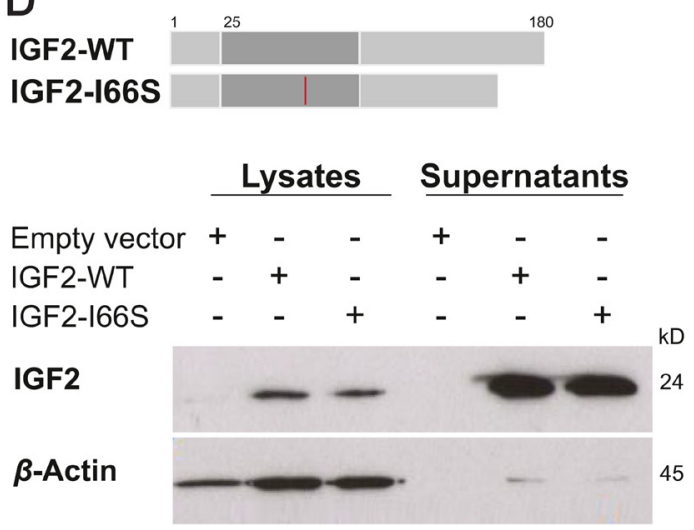

B

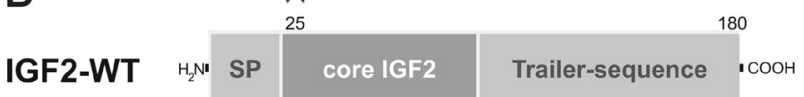

pro-IGF2

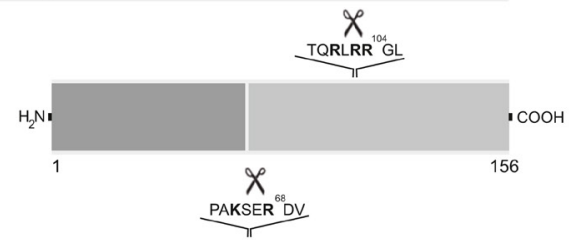

big IGF2

\section{Mature \\ IGF2-WT}

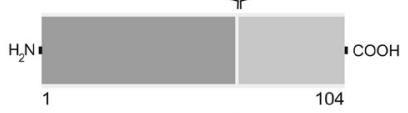

core IGF2 $\mathrm{COOH}$

C

IGF2-I66S

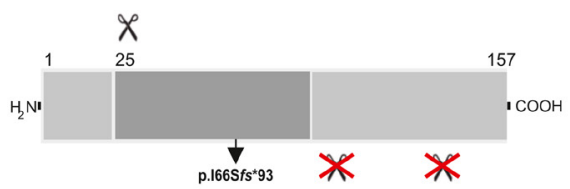

Mature IGF2-I66S

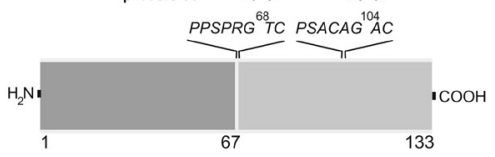

E

F
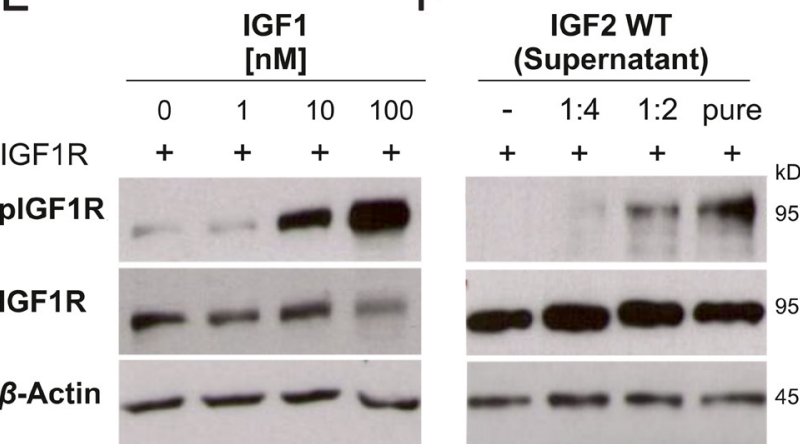

\section{Figure 3}

Illustration of potential conformational changes in p.I66S protein structure. (A) Schematic diagram of IGF2 maturation. IGF2 encodes an inactive 180-aa precursor protein which is post-translationally cleaved into a 67 -aa bioactive protein. First, the terminal signal peptide is proteolytically removed creating a 156-aa sequence. In the next step, the trailer sequence is proteolytically cleaved at two separated sites (TQRLRR ${ }^{104}$ and PAKSER ${ }^{68}$ ) generating the bioactive protein. (B) Illustration of p.I66S protein structure. Sequence analyses of the cleavage sites indicated a changed aa sequence at basic residues in the mutant: TQRLRR $\rightarrow$ PSACAG and PAKSER $\rightarrow$ PPSPRG. (C) Comparison of the WT (IGF2-WT, blue) and mutant (IGF2-166S, red) protein structure. 3D protein structure was predicted based on the crystal structure of IGF2-WT (pdb: 1/GL) using the iTASSER server and superpositioned with the WT structure. The mostly unstructured C-terminal extension due to the lack of posttranslational processing is illustrated. (D) Upper panel: Schematic presentation of IGF2 plasmids. The position of the c.195delC mutation is marked in red. Lower panel: Cell lysates and supernatants from transfected cells after immunoblotting with the indicated antibodies. (E) Whole-cell lysates from IGF1 stimulated (30 min) cells transfected with an IGF1R plasmid were subjected to immunoblotting using antibodies as indicated. (F) Whole-cell lysates were prepared from IGF1R transfected cells after stimulation (30 min) with IGF2-WT supernatants and immunoblotted for pIGF1R, total IGF1R and $\beta$-actin as loading control. Representative blot out of three independent experiments is shown. 
A

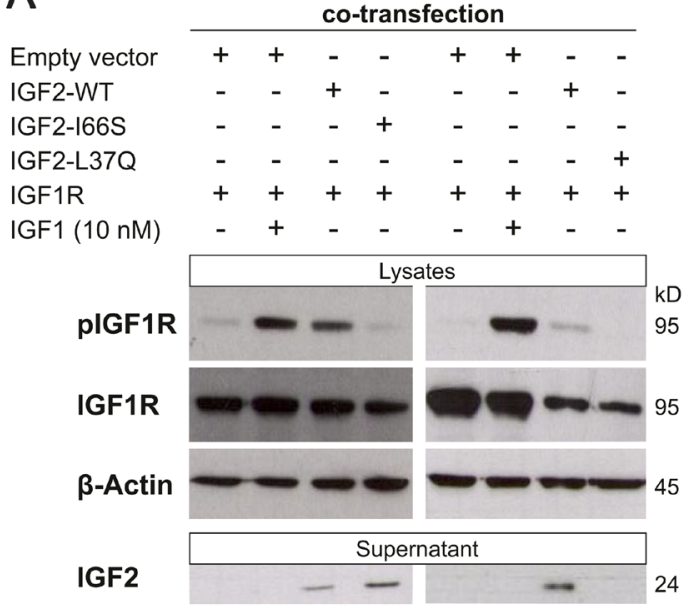

C

Empty vector IGF2-WT IGF2-I66S IGF2-L37Q IGF1R

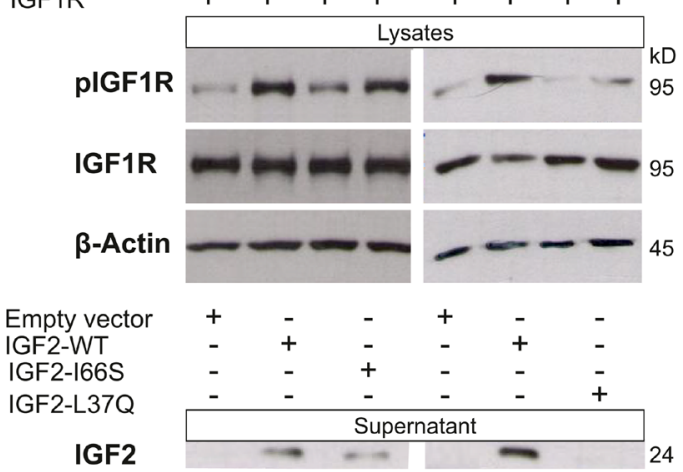

D

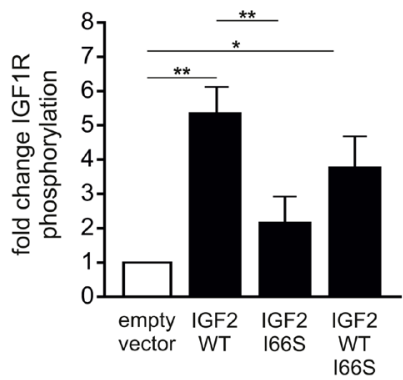

F

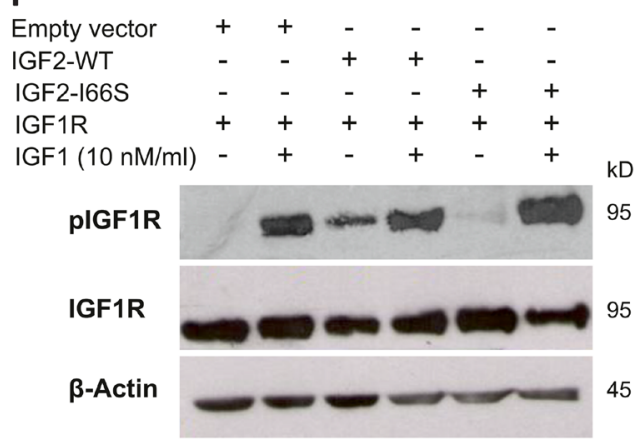

\section{Figure 4}

The p.166S variant inhibits IGF1R activation. (A) Immunoblots of untreated or rhIGF1 treated (30 min, $10 \mathrm{nM}$ ) cell lysates after co-transfection with IGF1R and IGF2 constructs were analyzed using the antibodies as indicated. In addition, supernatants from every approach were analyzed using an IGF2 antibody. (B) Densitometric evaluation of IGF1R activation (fold change of the variant compared to empty vector). pIGF1R levels were normalized to total IGF1R and $\beta$-actin protein amounts and results are shown as mean \pm S.E.M. Significant $P$-values are indicated. $* * * P<0.001$. (C) Cell lysates or supernatant from IGF1R transfected cells after stimulation with IGF2 supernatants (30 min) analyzed with the indicated antibodies. (D) Densitometric evaluation of IGF1 R activation (fold change of the variants compared to an empty vector control). $* P<0.05, * * P<0.01$. (E) Illustration of IGF2 residues interacting with domains of the IGF1 receptor according to Vashisth et al. (20). L1 residues are shown in red, CR residues in green, L2 residues in blue, and (F1-F2)' residues are shown in yellow. The IGF2 variant lacks L1, L2 and (F1-F2)' residues (highlighted in bold). (F) Rescue experiment with the p.166S variation. Lysates of IGF1R/IGF2 co-transfected HEK293 cells after stimulation with recombinant IGF1 (30 min, $10 \mathrm{nM}$ ) in comparison to unstimulated approaches. For each approach a representative Western blot out of three independent experiments is shown. 
all patients demonstrated decreased IGF2 serum levels. Two frameshift mutations satisfied the condition for nonsense-mediated mRNA decay that might explain the reduced serum concentrations in those patients. At this point the question arises, why we observed decreased IGF2 serum levels, although the IGF2 immunoassay detects mutant protein. It has to be mentioned that circulating IGFs are avidly bound to IGFBPs, in order to increase functional stability (27). The principal carrier of IGF1 and IGF2 in serum is IGFBP3 (28). Our IGF2 mutant showed a disturbed posttranslational processing, which is important for binary complex formation with IGFBPs in serum (29). The impaired interaction with IGFBP3 might induce a decreased IGF2 half-life time. Interestingly, we also observed decreased IGFBP3 serum levels in our patient; however, it remains unclear whether there is causal link between low IGF2 and IGFBP3 serum levels. It is conceivable that due to the reduced IGF2 levels, IGFBP3 might not have sufficient interaction partners for binary complex formation and will therefore rapidly cleared from the circulation (30). In addition, it has been shown that IGFBP3, are regulated by age, nutrition and GH (31). Our patient showed severe feeding difficulties leading to a pronounced underweight which might influence IGFBP3 availability.

Sequence analyses indicated functional consequences on protein structure through the IGF2 mutation, leading to a truncated protein $(52 \%$ of the WT precursor protein). However, in silico analysis predicated an enlarged IGF2 mutant protein, probably due to the disturbed posttranslational processing. IGF2 maturation begins with cleavage of the signal peptide, followed by glycosylation of the 89-residue trailer sequence that promote further cleavage at two crucial aa-cleavage sites: TQRLRR ${ }^{104}$ and PAKSER ${ }^{68}$ (32). The p.I66S variation induces a frameshift which alters both cleavage sites. Next, we analyzed whether the altered protein structure influence IGF2 activity. The growth-promoting effect of IGF2 resulted from its interaction with IGF1R, suggesting that the p.I66S variant is functionally not able to mediate IGF2/IGF1R signaling. In overexpression experiments, we demonstrated that mutant IGF2 plasmids showed a diminished IGF1R activation capacity. Furthermore, we can rescue pIGF1R in the mutant after stimulation with rhIGF1, indicating that the p.I66S variant is not able to bind to the IGF1 receptor. Vashisth et al. identified different IGF2/IGF1R-binding residues (20). Due to the frameshift, the mutant lacks C-terminal-binding residues including the binding site V43. Interestingly, it has been shown that V43 is essential for IGF2/IGF1R interaction, with $\geq 10$-fold decreases in binding affinities after removal (33). Given the fact that we can rescue IGF1R activation in the mutant after rhIGF1 stimulation; the question arises why IGF1 is not able to rescue IGF1R activation in vivo? In fact, different reasons come into consideration: (i) First, abnormal fetal growth in a sensitive period of development could have implications for health later in life with negative effects on physiology and metabolism (34). To what extent this can be assumed is questionable, given the severity of the postnatal phenotype and the fact that no catch-up growth can be observed at any time. (ii) Furthermore, several studies demonstrated that GH, IGF1 and IGF2 have independent and differential functions, indicating that they exert additive and synergistic effects $(35,36)$. Therefore, IGF1 and IGF2 might have synergistic effects using different pathways, meaning that IGF1R activation is not the essential pathway for the IGF2 growthpromoting effect. However, this requires the involvement of IGF2 in postnatal growth regulation, which is in contrast to previous assumptions. Interestingly, human IGF2 serum levels increase considerably during the first year of life with highest peak in adulthood $(\sim 700 \mathrm{ng} / \mathrm{mL}$, two times that of IGF1) (14). This increase is mainly due to the activation of the silent allele following birth, induced by the use of the biallelically expressed $\mathrm{P} 1$ promoter in the liver, which is unique to humans (37). During embryonic/ fetal and early postnatal development IGF2 transcription is monoallelically expressed from promoters P2-P4, with P4 being predominantly active $(3,38)$. To what extent IGF2 is important for postnatal growth remains subject of further investigations.

In summary, our patient has an IGF2 deficiency due to a biologically inactive IGF2 protein which is associated with SRS-like phenotype. The results of this study in combination with previous reports indicate that the p.I66S variant might be responsible for the pathogenic phenotype of the patient, which argues for the inclusion of IGF2 in gene panels designed for routine diagnostics of intrauterine growth failure without postnatal catch-up growth.

\section{Declaration of interest}

R P has received lecture fees from Novo Nordisk, Ferring, Sandoz, Lilly and Merck Serono.

\section{Funding}

This research did not receive any specific grant from any funding agency in the public, commercial or not-for-profit sector. J T H was supported by the Fonds der Chemischen Industrie (Material Cost Allowance). 


\section{Authors contribution statement}

D R and H P performed the experimental work. J R L, R A J coordinated, performed and analyzed NGS sequencing; $D \mathrm{R}$ and $\mathrm{H} P$ coordinated, performed and analyzed targeted sequencing. R P, J K, F S S, and W K collected clinical data and R P provided patient material. J T H performed structural modeling analyses. D L D, F R, J T H, J K, W K, R A J and R P contributed to the manuscript. R P coordinated the project.

\section{Acknowledgement}

The authors thank the participating family for their kind cooperation.

\section{References}

1 LeRoith D \& Yakar S. Mechanisms of disease: metabolic effects of growth hormone and insulin-like growth factor 1. Nature Clinical Practice Endocrinology and Metabolism 20073 302-310. (https://doi. org/10.1038/ncpendmet0427)

2 Baxter RC. Insulin-like growth factor (IGF)-binding proteins: interactions with IGFs and intrinsic bioactivities. American Journal of Physiology: Endocrinology and Metabolism 2000278 E967-E976. (https://doi.org/10.1152/ajpendo.2000.278.6.E967)

3 Engström W, Shokrai A, Otte K, Granérus M, Gessbo A, Bierke P, Madej A, Sjölund M \& Ward A. Transcriptional regulation and biological significance of the insulin like growth factor II gene. Cell Proliferation 199831 173-189. (https://doi. org/10.1111/j.1365-2184.1998.tb01196.x)

4 Bergman D, Halje M, Nordin M \& Engström W. Insulin-like growth factor 2 in development and disease: a mini-review. Gerontology 2013 59 240-249. (https://doi.org/10.1159/000343995)

5 DeChiara TM, Robertson EJ \& Efstratiadis A. Parental imprinting of the mouse insulin-like growth factor II gene. Cell 199164 849-859. (https://doi.org/10.1016/0092-8674(91)90513-X)

6 Demars J \& Gicquel C. Epigenetic and genetic disturbance of the imprinted 11p15 region in Beckwith-Wiedemann and SilverRussell syndromes. Clinical Genetics 201281 350-361. (https://doi. org/10.1111/j.1399-0004.2011.01822.x)

7 Gicquel C, Rossignol S, Cabrol S, Houang M, Steunou V, Barbu V, Danton F, Thibaud N, Le Merrer M, Burglen L et al. Epimutation of the telomeric imprinting center region on chromosome $11 \mathrm{p} 15$ in Silver-Russell syndrome. Nature Genetics 200537 1003-1007. (https:// doi.org/10.1038/ng1629)

8 Saal HM. Russell-Silver syndrome. In GeneReviews. Seattle: University of Washington, 1993.

9 Begemann M, Zirn B, Santen G, Wirthgen E, Soellner L, Büttel HM, Schweizer R, van Workum W, Binder G \& Eggermann T. Paternally inherited IGF2 mutation and growth restriction. New England Journal of Medicine 2015373 349-356. (https://doi.org/10.1056/ NEJMoa1415227)

10 Abi Habib W, Brioude F, Edouard T, Bennett JT, Lienhardt-Roussie A, Tixier F, Salem J, Yuen T, Azzi S, Le Bouc Y et al. Genetic disruption of the oncogenic HMGA2-PLAG1-IGF2 pathway causes fetal growth restriction. Genetics in Medicine 201820 250-258. (https://doi. org/10.1038/gim.2017.105)

11 Yamoto K, Saitsu H, Nakagawa N, Nakajima H, Hasegawa T, Fujisawa Y, Kagami M, Fukami M \& Ogata T. De novo IGF2 mutation on the paternal allele in a patient with Silver-Russell syndrome and ectrodactyly. Human Mutation 201738 953-958. (https://doi. org/10.1002/humu.23253)

12 Liu D, Wang Y, Yang XA \& Liu D. De novo mutation of paternal IGF2 gene causing Silver-Russell syndrome in a sporadic patient. Frontiers in Genetics 20178 105. (https://doi.org/10.3389/fgene.2017.00105)

13 Melchers P \& Preuss U. Revision of the Kaufman assessment battery for children for German speaking regions. Part 2: areas of application and criteria of reliability. Zeitschrift fur Kinder- und Jugendpsychiatrie $199220223-231$.

14 Blum WF, Ranke MB \& Bierich JR. A specific radioimmunoassay for insulin-like growth factor II: the interference of IGF binding proteins can be blocked by excess IGF-I. Acta Endocrinologica 1988118 374-380. (https://doi.org/10.1530/acta.0.1180374)

15 Zhang Y. I-TASSER server for protein 3D structure prediction. BMC Bioinformatics 20089 40. (https://doi.org/10.1186/1471-2105-9-40)

16 Dransfield DT, Cohen EH, Chang Q, Sparrow LG, Bentley JD, Dolezal O, Xiao X, Peat TS, Newman J, Pilling PA et al. A human monoclonal antibody against insulin-like growth factor-II blocks the growth of human hepatocellular carcinoma cell lines in vitro and in vivo. Molecular Cancer Therapeutics 20109 1809-1819. (https://doi. org/10.1158/1535-7163.MCT-09-1134)

17 Wakeling EL, Brioude F, Lokulo-Sodipe O, O'Connell SM, Salem J, Bliek J, Canton APM, Chrzanowska KH, Davies JH, Dias RP et al. Diagnosis and management of Silver-Russell syndrome: first international consensus statement. Nature Reviews Endocrinology 2017 13 105-124. (https://doi.org/10.1038/nrendo.2016.138)

18 Pagter-Holthuizen P de, Höppener JW, Jansen M, Geurts van Kessel AH, van Ommen GJ \& Sussenbach JS. Chromosomal localization and preliminary characterization of the human gene encoding insulin-like growth factor II. Human Genetics 198569 170-173. (https://doi.org/10.1007/BF00293291)

19 Qiu Q, Basak A, Mbikay M, Tsang BK \& Gruslin A. Role of proIGF-II processing by proprotein convertase 4 in human placental development. PNAS 2005102 11047-11052. (https://doi. org/10.1073/pnas.0502357102)

20 Vashisth $\mathrm{H} \&$ Abrams CF. All-atom structural models for complexes of insulin-like growth factors IGF1 and IGF2 with their cognate receptor. Journal of Molecular Biology 2010400 645-658. (https://doi. org/10.1016/j.jmb.2010.05.025)

21 Liu JP, Baker J, Perkins AS, Robertson EJ \& Efstratiadis A. Mice carrying null mutations of the genes encoding insulin-like growth factor I (Igf-1) and type 1 IGF receptor (Igf1r). Cell 199375 59-72.

22 Powell-Braxton L, Hollingshead P, Warburton C, Dowd M, PittsMeek S, Dalton D, Gillett N \& Stewart TA. IGF-I is required for normal embryonic growth in mice. Genes and Development 19937 2609-2617. (https://doi.org/10.1101/gad.7.12b.2609)

23 Netchine I, Azzi S, Le Bouc Y \& Savage MO. IGF1 molecular anomalies demonstrate its critical role in fetal, postnatal growth and brain development. Best Practice and Research Clinical Endocrinology and Metabolism 201125 181-190. (https://doi.org/10.1016/j. beem.2010.08.005)

24 DeChiara TM, Efstratiadis A \& Robertsen EJ. A growth-deficiency phenotype in heterozygous mice carrying an insulin-like growth factor II gene disrupted by targeting. Nature 1990345 78-80. (https://doi.org/10.1038/345078a0)

25 Verhaeghe J, Van Bree R, Van Herck E, Laureys J, Bouillon R \& Van Assche FA. C-peptide, insulin-like growth factors I and II, and insulin-like growth factor binding protein-1 in umbilical cord serum: correlations with birth weight. American Journal of Obstetrics and Gynecology 1993169 89-97. (https://doi.org/10.1016/00029378(93)90137-8)

26 Constância M, Hemberger M, Hughes J, Dean W, Ferguson-Smith A Fundele R, Stewart F, Kelsey G, Fowden A, Sibley C et al. Placentalspecific IGF-II is a major modulator of placental and fetal growth. Nature 2002417 945-948. (https://doi.org/10.1038/nature00819)

27 Clemmons DR. Insulin-like growth factor binding proteins and their role in controlling IGF actions. Cytokine and Growth Factor Reviews 19978 45-62. (https://doi.org/10.1016/S13596101(96)00053-6)

28 Baxter RC \& Martin JL. Binding proteins for the insulin-like growth factors: structure, regulation and function. Progress in Growth Factor Research 19891 49-68. (https://doi.org/10.1016/09552235(89)90041-0) 
29 Qiu Q, Yan X, Bell M, Di J, Tsang BK \& Gruslin A. Mature IGF-II prevents the formation of 'big' IGF-II/IGFBP-2 complex in the human circulation. Growth Hormone and IGF Research 201020 110-117. (https://doi.org/10.1016/j.ghir.2009.11.001)

30 Hodgkinson SC, Davis SR, Moore LG, Henderson HV \& Gluckman PD. Metabolic clearance of insulin-like growth factor-II in sheep. Journal of Endocrinology 1989123 461-468. (https://doi. org/10.1677/joe.0.1230461)

31 Livingstone C \& Borai A. Insulin-like growth factor-II: its role in metabolic and endocrine disease. Clinical Endocrinology 201480 773-781. (https://doi.org/10.1111/cen.12446)

32 Daughaday WH, Trivedi B \& Baxter RC. Serum 'big insulin-like growth factor II'; from patients with tumor hypoglycemia lacks normal E-domain O-linked glycosylation, a possible determinant of normal propeptide processing. PNAS 199390 5823-5827. (https:// doi.org/10.1073/pnas.90.12.5823)

33 Alvino CL, McNeil KA, Ong SC, Delaine C, Booker GW, Wallace JC, Whittaker J \& Forbes BE. A novel approach to identify two distinct receptor binding surfaces of insulin-like growth factor II. Journal of
Biological Chemistry 2009284 7656-7664. (https://doi.org/10.1074/ jbc.M808061200)

34 Godfrey KM \& Barker DJ. Fetal programming and adult health. Public Health Nutrition 20014 611-624. (https://doi.org/10.1079/PHN2001145)

35 Lupu F, Terwilliger JD, Lee K, Segre GV \& Efstratiadis A. Roles of growth hormone and insulin-like growth factor 1 in mouse postnatal growth. Developmental Biology 2001229 141-162. (https://doi. org/10.1006/dbio.2000.9975)

36 Wu S, Yang W \& De Luca F. Insulin-like growth factor-independent effects of growth hormone on growth plate chondrogenesis and longitudinal bone growth. Endocrinology 2015156 2541-2551. (https://doi.org/10.1210/en.2014-1983)

37 Ekström TJ, Cui H, Li X \& Ohlsson R. Promoter-specific IGF2 imprinting status and its plasticity during human liver development. Development 1995121 309-316.

38 Ekström TJ, Cui H, Nyström A, Rutanen EM \& Ohlsson R. Monoallelic expression of IGF2 at the human fetal/maternal boundary. Molecular Reproduction and Development 199541 177-183. (https://doi.org/10.1002/mrd.1080410208)

Received 8 August 2018

Revised version received 5 October 2018

Accepted 9 October 2018 